\title{
DYRK1A up-regulation specifically impairs a presynaptic form of long-term potentiation.
}

Aude-Marie Lepagnol-Bestel ${ }^{1}$, Simon Haziza ${ }^{1,2, a}$, Julia Viard ${ }^{1}$, Paul A. Salin ${ }^{3}$, Valérie Crépel ${ }^{4}$, Arnaud Duchon ${ }^{5,6,7,8,9}$, Yann Herault ${ }^{4,5,6,7,8,9}$, Michel Simonneau' ${ }^{1,2,10}$

\footnotetext{
${ }^{1}$ Centre Psychiatrie \& Neurosciences, INSERM U894, 75014 Paris, France.

${ }^{2}$ Laboratoire Aimé Cotton, CNRS, Univ. Paris-Sud, ENS Paris-Saclay, Université Paris-Saclay, 91405 Orsay, France.

${ }^{3}$ UMR CNRS 5167, Lyon, France.

${ }^{4}$ Mediterranean Institute of Neurobiology, INSERM, Marseille, France; Aix-Marseille University, Marseille, France.

${ }^{5}$ Institut de Génétique et de Biologie Moléculaire et Cellulaire, Illkirch, France.

${ }^{6}$ CNRS, UMR7104, Illkirch, France.

${ }^{7}$ INSERM, U964, Illkirch, France.

${ }^{8}$ Université de Strasbourg, Illkirch, France.

${ }^{9}$ PHENOMIN, Institut Clinique de la Souris, ICS; GIE CERBM, CNRS, INSERM, University of Strasbourg, 1 rue Laurent Fries, F67404 Illkirch-Graffenstaden, France.

${ }^{10}$ Department of Biology, Ecole Normale Supérieure de Paris-Saclay, Université Paris-Saclay, 94230 Cachan, France.

a Present address: James H. Clark Center, Stanford University, Stanford, CA, USA

Correspondence: Simonneau M (michel.simonneau@ens-paris-saclay.fr).
}

\section{ABSTRACT}

Chromosome 21 DYRK1A kinase has long been associated with a variety of psychiatric diseases including Down Syndrome. We previously showed that Dyrk1A interacts with SWI/SNF (SWItch/Sucrose Non-Fermentable) nucleosome remodeling complex inducing expression changes of genes encoding key neuronal proteins. However, the functional impact of this kinase at the synapse level remains unclear. We studied a mouse model that incorporated the YAC 152F7 (570 kb) encoding six chromosome 21 genes including DYRK1A. We found that DYRK1A Interacts with the key chromatin remodelers EP300 and CREBBP. Moreover, we observed changes in the transcriptional levels of genes encoding presynaptic proteins involved in glutamate vesicle exocytosis, namely Rims1, Munc13-1, Syn2, Rab3A. This result prompted us to investigate the two main forms of long-term potentiation (LTP) required for learning and memory: the ( $\mathrm{N}$-methyl $\mathrm{d}$-aspartate) receptor-dependent postsynaptic form versus the glutamate release-dependent presynaptic form. Interestingly, extracellular electrophysiological recordings in hippocampal slices of the YAC mouse line revealed that only the presynaptic forms of plasticity were impacted, leaving the post-synaptic form of plasticity intact. To refine our findings, we used a mouse BAC 189N3 (152 kb) line that only triplicate the gene Dyrk1A. Again, we found that this presynaptic form of LTP is also impaired in this mouse line. This result demonstrates that abnormal up-regulation of Dyrk $1 \mathrm{~A}$ alone is sufficient to inhibit specifically the presynaptic forms of LTP. Altogether, our results suggest that impairment of DYRK1A gene dosage may impact memory precision, and therefore reinforce our mechanistic understanding of the cognitive impairment detected in this mouse model. 


\section{INTRODUCTION}

Down syndrome (DS), a human genetic disorder, is the most common form of intellectual disability (ID), affecting $1 / 1,000$ births. DS is caused by the presence of a third copy of up to 234 genes from Homo sapiens autosome 21 (Hsa21) (1). Despite a broad spectrum of clinical symptoms, virtually all people affected by DS develop Alzheimer's disease pathology by 40 years of age, with intellectual deficits that impair learning and memory (1-4). However, a detailed understanding of the precise contribution of each Hsa21 gene to the cognitive impairment found in DS patients is still lacking.

Chromosome 21 Dyrk1A (dual-specificity tyrosine phosphorylated and regulated kinase 1A) gene is a major player in DS whom overexpression impacts the synaptic plasticity within the hippocampus and the prefrontal cortex (5-7). Converging evidence suggests that DYRK1A might be critical for learning and memory processes $(8,9)$. However, a clear understanding of the regulatory pathways impaired by a trisomy of DYRK1A is still lacking.

Here, we used two DS mouse models. The first model is a human YAC mouse model (152F7 line) that displays a $\sim 570 \mathrm{~kb}$ human genomic region surrounding the DYRK1A gene and including five other genes (10). The second is a Dyrk1A BAC model (189N3 line) that gives a triplication of $\sim 152 \mathrm{~kb}$ mouse Dyrk1a locus (6). By performing exome sequencing and mass spectroscopy in these mouse models, we recently uncovered two deregulated repertoires associated with chromatin and synaptic pathways (11). Interestingly, several studies reported that these lines display behavioral defects in episodic-like forms of memory $(10,12)$ Indeed, both 152F7 and 189N3 mouse models showed impairment in long-term spatial memory tested by Morris Water Maze $(10,12,13)$ or Novel Object Recognition task (14). These results indicate that both the "where" and the "what" pathways of episodic memory (15) are impacted in these models. These two pathways involve respectively the Lateral Entorhinal Cortex (LEC) that encodes experience from a first-person (egocentric) perspective, and the Medial Entorhinal Cortex (MEC) to the world-centered (allocentric) coding of place cells, grid cells, and head direction cells (16). Both LEC and MEC pathways share the same Dentate Gyrus-CA3 (Mossy Fiber-CA3) and CA3-CA1 pathways (15). In spite of these defects in both "where" and "what" hippocampal pathways, no study of long-term potentiation (LTP), a prominent cellular model thought to underlie long-term memory, have been reported for both 152F7 and 189N3 mouse lines $(6,10)$.

In this paper, our aim was to evidence a relation between these cognitive defects and a functional synaptic defect in these 152F7 and 189N3 mouse lines. Here, we first show here that DYRK1A directly interacts with two chromatin remodelers in neuron nuclei: EP300 and CREBBP. Moreover, using in situ hybridization, we found that deregulation of DYRK1A dosage indirectly induced changes in expression of genes encoding pre-synaptic proteins. Altogether, 
these results prompted us to investigate whether the synaptic plasticity is impaired in the Dentate Gyrus Mossy Fiber-CA3 pathway that is known to involve a presynaptic form of LTP linked to presynaptic proteins $(17,18)$. Therefore, using extracellular field recording in hippocampal slices, we found that the NMDA-independent long-term potentiation of the Dentate Gyrus-CA3 synapses is specifically impaired in these two mouse lines. These results reveal a previously unappreciated impact of Hsa21 DYRK1A on synaptic plasticity with possible consequences for a variety of neuropsychiatric diseases as diverse as Intellectual Disability, Late-Onset-Alzheimer Disease and Autism Spectrum Disorders. 


\section{RESULTS}

\section{Hsa21 DYRK1A interacts with the chromatin remodelers CREBBP and EP300}

Figure 1. Interaction of HSA21 DYRK1A with chromatin remodelers

Supplementary Figure 1. Schematic representation of the human YAC 152F7 transgene and of the mouse BAC 189N3 transgene.

Supplementary Figure 2. Schematic representation of the mouse genomic region syntenic of the human YAC 152F7 transgene and that includes the mouse BAC 189N3 transgene.

Supplementary Figure 3. Nuclear protein-protein interactions and deregulation of interaction network in transgenic mouse models.

The 152F7 mouse model incorporates $570 \mathrm{~kb}$ fragment of Hsa21 with six protein-coding genes including DYRK1A (Supplementary Figure 1). One of the six genes, DSCR9 is a primatespecific gene (19). We recently evidenced (11) that DSCR9 directly interacts with clusterin, a genetic risk factor of Late-Onset Alzheimer disease (20). The syntenic region in mouse chromosome 16 genome is more condensed with $\sim 350 \mathrm{~kb}$ (Supplementary Figure 2) instead of $\sim 570 \mathrm{~kb}$ in Hsa21. The organization of genes is similar between human and mouse genome with the sequence 3' to 5': Ripply3, Pigp, TTC3, Vps26c and Dyrk1A.

Here, we first analyzed protein-protein interactions in order to unravel possible molecular changes involved in cognition. We recently uncovered DS-related protein-protein interaction (PPI) map using a high-throughput, domain-based yeast two-hybrid (Y2H) technology against a human brain library (11). We attained more than $1200 \mathrm{PPI}$, among which we identified direct interactions between DYRK1A and EP300 or CREBBP (11). This result is consistent with previous findings using mass spectrometry methodologies (21). EP300 and CREBPP are two members of the p300-CBP coactivator family containing a histone acetyltransferase (HAT) domain involved in chromatin remodeling (22). Mutations in these genes have been shown to cause Rubinstein-Taybi Syndrome (23). To further validate these two interactions, we performed immunoprecipitation (IP) on HEK293 cells using anti-EP300 and anti-CREBBP antibodies and successfully identified DYRK1A (Figure 1A-B). We next validated direct nuclear interactions of Dyrk1A with EP300 and CREBBP in cortical neurons in vitro using a proximity ligation assay (PLA) (Figure 1C and Supplementary Figure 3).

Quantification of these interactions in 189N3 transgenic neurons compared to wild-type neurons indicate a statistically significant increase in DYRK1A-EP300 and DYRK1A-CREBBP interactions (Figure 1D). In contrast, we found no changes in the number of EP300-SMARCA2 and CREBBP-SMARCA2 interactions between 189N3 transgenic neurons compared to wildtype neurons (Figure 1D and Supplementary Figure 3). These results suggest that this increase in the number of DYRK1A-EP300 and DYRK1A-CREBBP in 189N3 can induce changes in transcriptome of neurons. Quantitative In Situ Hybridization (ISH) from Allen Brain 
Atlas indicates that both Dyrk1a, Ep300 and Crebbp are highly expressed in adult mouse hippocampus (Figure 1E). Altogether, these results identify chromatin regulators as direct interactors of DYRK1A.

Dyrk1A up-regulation affects the transcription of genes encoding presynaptic proteins Figure 2. Presynaptic protein expression in the adult 152F7 mouse hippocampus as compared to control mice.

Supplementary Figure 4. Quantification of Rims1 RNA in WT and $152 F 7$ hippocampus using quantitative In Situ Hybridation

Supplementary Figure 5. Comparison of transcripts encoding presynaptic proteins using either Q-RT PCR from laser assisted microdissection or RNAseq single cell transcriptomics

As EP300 and CREBBP encode HATs and impact chromatin remodeling, we hypothesized that changes in the level of DYRK1A would affect its interactions with these HAT partners and would consequently modify the transcription of genes involved in synaptic function (24-27). We tested whether genes encoding presynaptic proteins were deregulated in 152F7 hippocampal sub-regions. We first quantified Rims1 transcriptional changes using quantitative radioactive ISH on hippocampal sections of 152F7 and wild type (WT) mice. We detected a significant decrease in Rims1 transcript in both DG, CA3 and CA1 (Supplementary Figure 4). Both Rim1, Rab3a, Rims1 and Munc13-1 are involved in the presynaptic release of glutamate (28) (Figure 2A). Synapsin I and synapsin II are widely expressed synaptic vesicle phosphoproteins that have been proposed to play an important role in synaptic transmission and short-term synaptic plasticity $(29,30)$. We first used Western blotting of Rim1, Rab3a, Munc13-1 and Synapsin 2A proteins from hippocampus extracts of 152F7 and WT mice. We found a decrease in Synapsin 2A protein for 152F7 as compared to WT mice (Figure 2B). We next used RT-Q-PCR following laser-assisted micro-dissection of hippocampal sub-regions (Figure 2C) to quantify possible changes of these transcripts between $152 \mathrm{~F} 7$ and control mice. We found that the protein levels of Rims1, Syn2, Munc13-1 and Rab3a decreased in 152F7 dentate gyrus compared to wild type (Figure 2D). We compared transcript levels for dorsal DG, CA3 and CA1 from our data obtained by Q-RT-PCR from laser-assisted microdissection with Hipposeq data obtained and from single-cell transcriptomics (31). Quantitative changes were fully coherent for Rims1 and partially for Syn2, not coherent for Rab3a and Munc13-1, suggesting 3-D heterogeneity (Supplementary Figure 5).

Altogether, these results show that change in Dyrk1a dosage can modify the expression of gene encoding proteins involved in the molecular mechanism of presynaptic vesicle release. 


\section{Dyrk1A up-regulation specifically impairs a presynaptic form of LTP at the DG-CA3 hippocampal synapse}

Figure 3. Presynaptic LTP between Dentate Gyrus mossy fibers and CA3 impaired in both adult 152 F7 and 189N3 mouse hippocampus.

Two main forms of LTP have been characterized in the mammalian brain. One requires activation of postsynaptic NMDA (N-methyl d-aspartate) receptors, whereas the other, as for mossy fiber (MF) LTP, is NMDAR independent and require presynaptic mechanisms. This presynaptic form of LTP at the MF-CA3 synapse have been widely characterized and requires activation of several presynaptic proteins linked to glutamate vesicle exocytosis such as Rims1, Munc13-1, Syn2, Rab3A $(17,28)$.

Here, we used field excitatory postsynaptic potential (fEPSP) to functionally test the effect of abnormal up-regulation of Dyrk1a in both mouse models $152 \mathrm{~F} 7$ and $189 \mathrm{~N}$. We recorded in the lucidum of CA3 and MF-mediated fEPSP were evoked by weak electrical stimulations in the dentate gyrus. We induced LTP by tetanus at $25 \mathrm{~Hz}$ during 5 s under NMDA blocker D-AP5 (D2-amino-5-phosphonovalerate) to prevent NMDA-dependent LTP from occurring (Figure $3 \mathbf{A}$ ). In both models, we found that this form of NMDA independent LTP was absent at the dentate gyrus-CA3 synapse (Figure 3 B-D), suggesting that Dyrk1A overexpression alone is sufficient to inhibit mossy fiber LTP. These results show that abnormal Dyrk1a dosage can modify the expression of genes encoding proteins involved in the molecular mechanism leading to presynaptic release. 


\section{DISCUSSION}

The general aim of this study was to evidence a relation between the cognitive defects and synapse plasticity impairments in the 152F7 Down syndrome mouse model (10), in which episodic spatial memory is impaired without noticeable defects in hippocampal synaptic plasticity $(10,13)$. The $152 F 7$ mouse model expresses a human YAC fragment of $570 \mathrm{~kb}$ that includes six distinct genes, among which two transcription regulators, RIPPLY3 and DYRK1A. RIPPLY3 was characterized as a transcriptional co-repressor but its putative function is far from being understood. In contrast, DYRK1A encodes a kinase with multiple subcellular sites of action including transcriptional regulation. We previously demonstrated that DYRK1A interacts with the REST/NRSF-SWI/SNF chromatin remodeling complex to deregulate gene clusters involved in the neuronal phenotypic traits of Down syndrome (32). Moreover, it was recently shown that the DYRK1A regulates transcription of a subset of genes by associating to their proximal promoter regions and phosphorylating the C-terminal domain of the RNA polymerase II (33). In this study, we found that DYRK1A directly interacts with chromatin remodelers EP300 and CREBPP that are known to impact synaptic plasticity (24).

Recent data suggest that memory precision defects can be generated by deregulation of genes involved in hippocampus Dentate Gyrus-CA3 connections, in particular Dentate Gyrus Mossy Fibers (MF)-CA3 synapse (18, 34-38). A During contextual fear memory consolidation, the strength of mossy fiber connections is selectively increased between dentate gyrus engram cells and CA3 engram cells (i.e. mossy fiber LTP-like process, Ryan et al, 2015). This interplay between the dentate gyrus and CA3 area is required for long-term memory process that requires pattern separation, a process that inhibits memory generalization. Increased time-dependent loss of memory precision is characterized by an overgeneralization of fear that is a feature of post-traumatic stress disorder (PTSD) (39). Memory imprecision also characterizes mild cognitive impairment $(\mathrm{MCl})$ that precedes Late-Onset Alzheimer Disease $(40,41)$. The connectivity between Dentate gyrus cells (DGC) and CA3 pyramidal neurons via DGC MF in the hippocampus is central for memory precision (32, 34). Hippocampal MF are the presynaptic component of MF-CA3 synapses that display both structural and functional plasticity in relation to the precision of hippocampus dependent memories. The earliest indication that large presynaptic boutons called large mossy fiber terminals (LMTs) have distinct physiological properties originated from the finding that the induction of LTP at LMTs was independent of NMDA receptor activation (42). Since MF- LTP was induced independently of activity on the postsynaptic site, it was suggested that this LTP was solely triggered by the presynaptic terminal itself (43). The presynaptic vesicle protein Rab3a and the active zone protein Rims1 and Munc13-1 have been shown to be required molecular components of the presynaptic mechanism underlying the maintenance and expression of MF-LTP in the hippocampus (44-47) 
From these results, we examined if $D Y R K 1 A$ gene dosage can modify the regulation of genes that encode proteins involved in presynapse vesicle exocytosis as knockout of the related genes disrupt MF-CA3 non-NMDA LTP. Using quantitative ISH, we were able to find a statistically significant decrease of Rims1 in 152F7 Dentate Gyrus. We identified significant decrease in transcripts level involved in presynapse vesicle exocytosis such as Rims1, Rab3a and Munc13-1 whose knockout induces impairment in MF-CA3 LTP. Other transcripts such as Syn2 involved in presynapse functioning (Spillane et al., 1995) were also impacted. Impairment of MF-CA3 LTP has been reported only for knockout of Rims1, Rab3 and Munc13-1 with no effect for haploinsufficiency (44-47). Here, we propose that the combined haploinsufficiency for Rims1, Rab3 and Munc13-1 can have a functional consequence similar to knockout of one of these genes, leading to a phenocopy of knockout.

Furthermore, we were able to demonstrate using the $189 \mathrm{~N} 3$ mouse model overexpressing only Dyrk1A that MF-CA3 LTP defect can be directly linked to Dyrk1A overexpression. Implication of DYRK1A in LTP defects that is linked to memory precision can be of clinical importance for Down Syndrome but also for Autism Spectrum Disorders as DYRK1A is one of the key candidate genes in this disease with a 20 fold increases in risk $(48,49)$. Altogether, this work identifies novel molecular and functional novel biomarkers that are involved in the cognitive signature displayed by mouse models of Down Syndrome. Our study thereby provides a novel mechanistic and potentially therapeutic understanding of deregulated signaling downstream of DYRK1A up-regulation. 


\section{ACKNOWLEGDMENTS}

This work was partly supported by European FP7-HEALTH AgedBrainSysbio and European JPND TransPathND program (to M.S.).

\section{REFERENCES}

1. Antonarakis,S.E. (2017) Down syndrome and the complexity of genome dosage imbalance. Nat. Rev. Genet., 18, 147-163.

2. Lott,I.T. and Head,E. (2019) Dementia in Down syndrome: unique insights for Alzheimer disease research. Nat. Rev. Neurol., 15, 135-147.

3. Dierssen,M. (2012) Down syndrome: the brain in trisomic mode. Nat. Rev. Neurosci., 13, 844-58.

4. Wiseman,F.K., Al-Janabi,T., Hardy,J., Karmiloff-Smith,A., Nizetic,D., Tybulewicz,V.L.J., Fisher,E.M.C. and Strydom,A. (2015) A genetic cause of Alzheimer disease: mechanistic insights from Down syndrome. Nat. Rev. Neurosci., 16, 564-574.

5. Ahn,K.-J., Jeong,H.K., Choi,H.-S., Ryoo,S.-R., Kim,Y.J., Goo,J.-S., Choi,S.-Y., Han,J.-S., Ha,I. and Song,W.-J. (2006) DYRK1A BAC transgenic mice show altered synaptic plasticity with learning and memory defects. Neurobiol. Dis., 22, 463-472.

6. Guedj,F., Pereira,P.L., Najas,S., Barallobre,M.-J., Chabert,C., Souchet,B., Sebrie,C., Verney,C., Herault,Y., Arbones,M., et al. (2012) DYRK1A: A master regulatory protein controlling brain growth. Neurobiol. Dis., 46, 190-203.

7. Thomazeau,A., Lassalle,O., lafrati,J., Souchet,B., Guedj,F., Janel,N., Chavis,P., Delabar,J. and Manzoni,O.J. (2014) Prefrontal Deficits in a Murine Model Overexpressing the Down Syndrome Candidate Gene Dyrk1a. J. Neurosci., 34, 1138-1147.

8. Jiang,X., Liu,C., Yu,T., Zhang,L., Meng,K., Xing,Z., Belichenko,P. V., Kleschevnikov,A.M., Pao,A., Peresie,J., et al. (2015) Genetic dissection of the Down syndrome critical region. Hum. Mol. Genet., 24, 6540-6551.

9. Arbones,M.L., Thomazeau,A., Nakano-Kobayashi,A., Hagiwara,M. and Delabar,J.M. (2019) DYRK1A and cognition: A lifelong relationship. Pharmacol. Ther., 194, 199-221.

10. Smith,D.J., Stevens,M.E., Sudanagunta,S.P., Bronson,R.T., Makhinson,M., Watabe,A.M., O'Dell,T.J., Fung,J., Weier,H.-U.G., Cheng,J.-F., et al. (1997) Functional screening of $2 \mathrm{Mb}$ of human chromosome 21q22.2 in transgenic mice implicates minibrain in learning defects associated with Down syndrome. Nat. Genet., 16, 28-36.

11. Viard,J., Loe-Mie,Y., Rachel Daudin, Khelfaoui,M., Christine Plancon, Anne Boland, Francisco Tejedor, Richard L. Huganir, Eunjoon Kim, Makoto Kinoshita, Guofa Liu, Volker Haucke, Thomas Moncion, Eugene Yu, Valérie Hindie,H.B., Mircher,C., Duchon,A., Herault,Y., Deleuze,J.-F., Rain,J.-C., et al. (2019) Chromosome 21 large-scale yeast two-hybrid screening coupled to proximity ligation assays uncovers novel interactors in dendritic spine linked to intellectual disabilities. Submitted.

12. Souchet,B., Guedj,F., Sahún,I., Duchon,A., Daubigney,F., Badel,A., Yanagawa,Y., Barallobre,M.J., Dierssen,M., Yu,E., et al. (2014) Excitation/inhibition balance and learning are modified by Dyrk1a gene dosage. Neurobiol. Dis., 69, 65-75.

13. Dierssen,M. (2012) Down syndrome: the brain in trisomic mode. Nat. Rev. Neurosci., 13, 844-858.

14. Nguyen,T.L., Duchon,A., Manousopoulou,A., Loaëc,N., Villiers,B., Pani,G., Karatas,M., Mechling,A.E., Harsan,L.-A., Limanton,E., et al. (2018) Correction of cognitive deficits in mouse models of Down syndrome by a pharmacological inhibitor of DYRK1A. Dis. Model. Mech., 11, dmm035634.

15. Knierim,J.J. (2015) The hippocampus. Curr. Biol., 25, R1116-R1121.

16. Wang,C., Chen,X., Lee,H., Deshmukh,S.S., Yoganarasimha,D., Savelli,F. and Knierim,J.J. (2018) Egocentric coding of external items in the lateral entorhinal cortex. Science, 362, 945-949. 
17. Castillo,P.E. (2012) Presynaptic LTP and LTD of Excitatory and Inhibitory Synapses. Cold Spring Harb. Perspect. Biol., 4, a005728-a005728.

18. Monday,H.R., Younts,T.J. and Castillo,P.E. (2018) Long-Term Plasticity of Neurotransmitter Release: Emerging Mechanisms and Contributions to Brain Function and Disease. Annu. Rev. Neurosci., 41, 299-322.

19. Takamatsu,K. (2002) Identification of Two Novel Primate-Specific Genes in DSCR. DNA Res., 9 , 89-97.

20. Lambert,J.C., Ibrahim-Verbaas,C. a, Harold,D., Naj, a C., Sims,R., Bellenguez,C., DeStafano, a L., Bis,J.C., Beecham,G.W., Grenier-Boley,B., et al. (2013) Meta-analysis of 74,046 individuals identifies 11 new susceptibility loci for Alzheimer's disease. Nat. Genet., 45, 1452-8.

21. Varjosalo,M., Keskitalo,S., Van Drogen,A., Nurkkala,H., Vichalkovski,A., Aebersold,R. and Gstaiger,M. (2013) The Protein Interaction Landscape of the Human CMGC Kinase Group. Cell Rep., 3, 1306-1320.

22. Goodman,R.H. and Smolik,S. (2000) CBP/p300 in cell growth, transformation, and development. Genes Dev., 14, 1553-77.

23. Coupry,I., Monnet,L., Attia,A.A.E.M., Taine,L., Lacombe,D. and Arveiler,B. (2004) Analysis of CBP (CREBBP) gene deletions in Rubinstein-Taybi syndrome patients using real-time quantitative PCR. Hum. Mutat., 23, 278-84.

24. Alarcón,J.M., Malleret,G., Touzani,K., Vronskaya,S., Ishii,S., Kandel,E.R. and Barco,A. (2004) Chromatin Acetylation, Memory, and LTP Are Impaired in CBP+/- Mice. Neuron, 42, 947-959.

25. Guan,Z., Giustetto,M., Lomvardas,S., Kim,J.-H., Miniaci,M.C., Schwartz,J.H., Thanos,D. and Kandel,E.R. (2002) Integration of Long-Term-Memory-Related Synaptic Plasticity Involves Bidirectional Regulation of Gene Expression and Chromatin Structure. Cell, 111, 483-493.

26. Kandel,E.R. (2001) The Molecular Biology of Memory Storage: A Dialogue Between Genes and Synapses. Science, 294, 1030-1038.

27. Levine,A.A., Guan,Z., Barco,A., Xu,S., Kandel,E.R. and Schwartz,J.H. (2005) CREB-binding protein controls response to cocaine by acetylating histones at the fosB promoter in the mouse striatum. Proc. Natl. Acad. Sci., 102, 19186-19191.

28. Acuna,C., Guo,Q., Burré,J., Sharma,M., Sun,J. and Südhof,T.C. (2014) Microsecond Dissection of Neurotransmitter Release: SNARE-Complex Assembly Dictates Speed and Ca2+ Sensitivity. Neuron, 82, 1088-1100.

29. Spillane,D.M., Rosahl,T.W., Südhof,T.C. and Malenka,R.C. (1995) Long-term potentiation in mice lacking synapsins. Neuropharmacology, 34, 1573-1579.

30. Cesca,F., Baldelli,P., Valtorta,F. and Benfenati,F. (2010) The synapsins: Key actors of synapse function and plasticity. Prog. Neurobiol., 91, 313-348.

31. Cembrowski,M.S., Wang,L., Sugino,K., Shields,B.C. and Spruston,N. (2016) Hipposeq: a comprehensive RNA-seq database of gene expression in hippocampal principal neurons. Elife, $\mathbf{5}$, e14997.

32. Lepagnol-Bestel,A.-M., Zvara,A., Maussion,G., Quignon,F., Ngimbous,B., Ramoz,N., Imbeaud,S., Loe-Mie,Y., Benihoud,K., Agier,N., et al. (2009) DYRK1A interacts with the REST/NRSF-SWI/SNF chromatin remodelling complex to deregulate gene clusters involved in the neuronal phenotypic traits of Down syndrome. Hum. Mol. Genet., 18, 1405-1414.

33. Vona,C.D., Bezdan,D., Islam,A.B.M.M.K., Salichs,E., López-Bigas,N., Ossowski,S. and Luna,S.D.L. (2015) Chromatin-wide profiling of DYRK1A reveals a role as a gene-specific RNA polymerase II CTD kinase. Mol. Cell, 10.1016/j.molcel.2014.12.026.

34. Guo,N., Soden,M.E., Herber,C., Kim,M.T., Besnard,A., Lin,P., Ma,X., Cepko,C.L., Zweifel,L.S. and Sahay,A. (2018) Dentate granule cell recruitment of feedforward inhibition governs engram maintenance and remote memory generalization. Nat. Med., 24, 438-449.

35. Lepagnol-Bestel,A.-M., Zvara,A., Maussion,G., Quignon,F., Ngimbous,B., Ramoz,N., Imbeaud,S., Loe-Mie,Y., Benihoud,K., Agier,N., et al. (2009) DYRK1A interacts with the REST/NRSF-SWI/SNF chromatin remodelling complex to deregulate gene clusters involved in the neuronal phenotypic 
traits of Down syndrome. Hum. Mol. Genet., 18, 1405-1414.

36. Jones,B.W., Deem,J., Younts,T.J., Weisenhaus,M., Sanford,C.A., Slack,M.C., Chin,J., Nachmanson,D., McKennon,A., Castillo,P.E., et al. (2016) Targeted deletion of AKAP7 in dentate granule cells impairs spatial discrimination. Elife, $\mathbf{5}$.

37. Weng,F.-J., Garcia,R.I., Lutzu,S., Alviña,K., Zhang,Y., Dushko,M., Ku,T., Zemoura,K., Rich,D., Garcia-Dominguez,D., et al. (2018) Npas4 Is a Critical Regulator of Learning-Induced Plasticity at Mossy Fiber-CA3 Synapses during Contextual Memory Formation. Neuron, 97, 1137-1152.e5.

38. Ryan,T.J., Roy,D.S., Pignatelli,M., Arons,A. and Tonegawa,S. (2015) Engram cells retain memory under retrograde amnesia. Science, 348, 1007-1013.

39. Maren,S., Phan,K.L. and Liberzon,I. (2013) The contextual brain: implications for fear conditioning, extinction and psychopathology. Nat. Rev. Neurosci., 14, 417-428.

40. Bakker,A., Krauss,G.L., Albert,M.S., Speck,C.L., Jones,L.R., Stark,C.E., Yassa,M.A., Bassett,S.S., Shelton,A.L. and Gallagher,M. (2012) Reduction of Hippocampal Hyperactivity Improves Cognition in Amnestic Mild Cognitive Impairment. Neuron, 74, 467-474.

41. Leal,S.L. and Yassa,M.A. (2015) Neurocognitive Aging and the Hippocampus across Species. Trends Neurosci., 38, 800-812.

42. Harris,E.W. and Cotman,C.W. (1986) Long-term potentiation of guinea pig mossy fiber responses is not blocked by N-methyl d-aspartate antagonists. Neurosci. Lett., 70, 132-137.

43. Zalutsky,R. and Nicoll,R. (1990) Comparison of two forms of long-term potentiation in single hippocampal neurons. Science, 248, 1619-1624.

44. Castillo,P.E., Janz,R., Sdhof,T.C., Tzounopoulos,T., Malenka,R.C. and Nicoll,R.A. (1997) Rab3A is essential for mossy fibre long-term potentiation in the hippocampus. Nature, 388, 590-593.

45. Castillo,P.E., Schoch,S., Schmitz,F., Südhof,T.C. and Malenka,R.C. (2002) RIM1 $\alpha$ is required for presynaptic long-term potentiation. Nature, 415, 327-330.

46. Lonart,G., Janz,R., Johnson,K.M. and Südhof,T.C. (1998) Mechanism of Action of rab3A in Mossy Fiber LTP. Neuron, 21, 1141-1150.

47. Yang,Y. and Calakos,N. (2011) Munc13-1 Is Required for Presynaptic Long-Term Potentiation. J. Neurosci., 31, 12053-12057.

48. Sanders,S.J., He,X., Willsey,A.J., Ercan-Sencicek,A.G., Samocha,K.E., Cicek,A.E., Murtha,M.T., Bal,V.H., Bishop,S.L., Dong,S., et al. (2015) Insights into Autism Spectrum Disorder Genomic Architecture and Biology from 71 Risk Loci. Neuron, 87, 1215-1233.

49. Willsey,A.J., Morris,M.T., Wang,S., Willsey,H.R., Sun,N., Teerikorpi,N., Baum,T.B., Cagney,G., Bender,K.J., Desai,T.A., et al. (2018) The Psychiatric Cell Map Initiative: A Convergent Systems Biological Approach to Illuminating Key Molecular Pathways in Neuropsychiatric Disorders. Cell, 174, 505-520. 


\section{MATERIALS AND METHODS}

\section{Animals and genotyping}

We used wild-type mice of the OF1 strain for neuronal primary culture, of the C57BL6 strain and $189 \mathrm{~N} 3$ or $\mathrm{Dp}(16) 1$ Yey transgenic line for neuronal primary cultures and electrophysiology and FVB strain and the 152F7 transgenic line for electrophysiology (10). Genotypes were determined using genomic DNA extracted from skeletal muscle fragments and the PCR protocol and primers as described previously (32).

\section{Quantitative In situ hybridization (ISH)}

For Rim1 in situ hybridization, a 1124 bp Rim1 PCR product (covering nucleotides 376 to 1499 of the XM_129709 cDNA sequence) was inserted into a pCRII-TOPO cloning vector) as described in. Xhol- or HindIII-linearized Rim1 inserts were used to generate antisense or sense [a35S]-rUTP (800 Ci/mmol, Amersham) labeled transcripts, using the P1460 riboprobe in vitro transcription systems (Promega), according to the manufacturer's instructions. Paraffinembedded coronal $15 \mu \mathrm{m}$ sections of P21 mouse brains, including hippocampal structures, were selected for ISH. Sections were hybridized with radiolabeled RNA probe (diluted to 105 $\mathrm{cpm} / \mu \mathrm{l}$ in $50 \%$ formamide hybridization buffer) in $50 \%$ formamide at $50^{\circ} \mathrm{C}$. Sections were successively washed in $50 \%$ formamide, $2 \times$ SSC, $10 \mathrm{mM}$ DTT at $65^{\circ} \mathrm{C}$, and then in increasingly stringent SSC washing solution, with a final wash at $0.1 \times S S C$ at $37^{\circ} \mathrm{C}$. The regional distribution of radioactivity was analyzed in the hippocampal region of wild-type and transgenic sections using a digital autoradiography imager (Micro Imager from Biospace Lab, Paris, France) and its analysis program (BetaVision, Biospace Lab).

\section{Protein extraction and Western blot analysis.}

HEK293 cells or mouse cortex (pool from three adult OF1 mice) were homogenized on ice in Tris-buffered saline (100 mM NaCl, $20 \mathrm{mM}$ Tris- $\mathrm{HCl}, \mathrm{pH} 7.4,1 \% \mathrm{NP} 40,1 \times \mathrm{CIP})$. The homogenates were centrifuged at $13,000 \mathrm{~g}$ for $10 \mathrm{~min}$ at $4^{\circ} \mathrm{C}$ and the supernatants were stored at $-80^{\circ} \mathrm{C}$. Cell lysate protein concentration was determined using the BCA Protein assay kit (ThermoFisher). For SDS-PAGE, 40 $\mu$ g of protein was diluted in Laemmli 1x (BioRad) with DTT and incubate for $5 \mathrm{mn}$ at $95^{\circ} \mathrm{C}$. Proteic samples were loaded in each lane of a $4-15 \%$ precast polyacrylamide gel (BioRad) and ran in Mini-Protean at $200 \mathrm{~V}$ in Tris/Glycine running buffer (BioRad). Following SDS-PAGE, proteins were semi-dry electroblotted onto nitrocellulose membranes using the Trans-Blot Turbo Transfer System (BioRad). Membranes were incubated for $1 \mathrm{~h}$ at room temperature in blocking solution (PBS $1 \mathrm{x}$ containing 5\% non-fat dried milk, $0.05 \%$ Tween 20 ) and then for overnight at $4^{\circ} \mathrm{C}$ with the primary antibody. Primary antibodies used were as shown in Supplementary Table S6. Membranes were washed in PBS $1 \mathrm{x}$ containing $0.05 \%$ tween 20 and incubated for $1 \mathrm{~h}$ at room temperature with anti-mouse, anti-rabbit or anti-goat HRP-conjugated secondary antibody. Membranes were washed three 
times in PBS $1 x$ containing $0.05 \%$ tween 20 . Immune complexes were visualized using the Clarity Western ECL Substrate (BioRad). Chemiluminescence was detected using the ChemiDoc XRS Imaging System (BioRad). Primary antibodies used were as shown in Supplementary Table S3. As secondary antibodies, we used protein A or protein G IgG, HRPconjugated whole antibody (1/5,000; Abcam ab7460 or ab7456 respectively).

\section{Laser-assisted microdissection, Total RNA preparation and quantitative real-time PCR (Q-RT-PCR) analysis}

Embryonic brain subregions were dissected as shown in Supplementary Figure. The left and right hippocampus was micro dissected from genotyped P21 mouse brains using a laserassisted capture microscope (Leica ASLMD instrument) with Leica polyethylene naphthalate membrane slides as described in. RNA preparation and Q-RT-PCR are performed as described in. Q-RT-PCR results are expressed in arbitrary unit.

\section{Electrophysiological analysis on 152F7 juvenile mice}

The methods used have been described elsewhere (Hanson et al., 2007). Briefly, for the preparation of hippocampal slices, 21-day-old mice were deeply anaesthetized with Nembutal. Brain slices $(300-400 \mu \mathrm{m})$ were cut in cold artificial cerebrospinal fluid (temperature $\approx 4$ $8^{\circ} \mathrm{C}$ ). The artificial cerebrospinal fluid (ACSF) contained $124 \mathrm{mM} \mathrm{NaCl}, 26 \mathrm{mM} \mathrm{NaHCO}$, $2.5 \mathrm{mM} \mathrm{KCl}, 1.25 \mathrm{mM} \mathrm{NaH} 2 \mathrm{PO} 4,2.5 \mathrm{mM} \mathrm{CaCl}$, $1.3 \mathrm{mM} \mathrm{MgCl}$, and $10 \mathrm{mM}$ glucose. Slices were maintained at room temperature for at least 1 hour in a submerged chamber containing ACSF equilibrated with 95\% O2 and 5\% CO2 and were then transferred to a super fusion chamber. Field EPSPs were recorded, using microelectrodes (1-3 M 2 ) filled with ACSF, at $22^{\circ}$ to $25^{\circ} \mathrm{C}$. Bipolar stainless steel electrodes were used for the electrical stimulation of Schaffer collaterals and mossy fibers $(0.1 \mathrm{~ms}, 10$ to $100 \mu \mathrm{A}$ pulses, intertrial intervals of 10 to $30 \mathrm{~s}$ ). Field EPSP recordings of mossy fibers and Schaffer collaterals were taken with a DAM80 amplifier (WPI), under visual control, with an upright microscope (BX50WI,Olympus). Mossy fiber LTP was induced by tetanus at $25 \mathrm{~Hz}$ for $5 \mathrm{~s}$, in the presence of $50 \mu \mathrm{M}$ D-AP5 (D (-) 2-amino-5-phosphonovaleric acid). Mossy fiber responses were identified in wild-type and 152F7 mice with the group 2-metabotropic glutamate receptor selective agonist DCG IV. The inhibitory effects of DCG IV $(10 \mu \mathrm{M})$ on mossy fiber inputs were similar in mutants and wildtype mice. NBQX $(5 \mu \mathrm{M})$ was applied at the end of each mossy fiber experiment, to assess the amplitude of fiber volleys. For Schaffer collateral LTP, stimulating and extracellular recording electrodes were placed in the stratum radiatum, and the GABA-A receptor antagonist picrotoxin $(100 \mu \mathrm{M})$ was added to the ACSF. In this series of experiments, the CA1 region was separated from the CA3 region by sectioning the brain slice with a knife before recording. Data were acquired and analyzed blind to genotype for LTP experiments. On- and offline data analyses were carried out with Acquis1-EIPhy software (developed by G. Sadoc, UNIC CNRS 
and ANVAR, France). Summary data are expressed as means \pm SEM. The following drugs were used: NBQX, D-APV, DCGIV (Tocris) and picrotoxin (Sigma).

\section{Electrophysiological analysis on 189 N3 adult mice}

All experiments were approved by the Institut National de la Santé et de la Recherche Médicale (INSERM) animal care and use agreement (D-13-055-19) and the European community council directive (2010/63/UE).

Preparation of Hippocampal Slices 189N3 KI and WT littermate male mice aged 17-22 week-old were shipped from IGBMC (Strasbourg, France) to INMED (Marseille, France). Mice were deeply anesthetized with xylazine $13 \mathrm{mg} / \mathrm{kg} / \mathrm{ketamine} 66 \mathrm{mg} / \mathrm{kg}$ and transcardially perfused with a modified artificial cerebrospinal fluid ( $\mathrm{mACSF}$ ) containing the following (in $\mathrm{mM}$ ): 132 choline, $2.5 \mathrm{KCl}, 1.25 \mathrm{NaH} 2 \mathrm{PO} 4,25 \mathrm{NaHCO}, 7 \mathrm{MgCl}$, $0.5 \mathrm{CaCl}$, and $8 \mathrm{D}$-glucose prior to decapitation. The brain was then removed rapidly, the hippocampi were dissected, and transverse $450 \mu \mathrm{M}$ thick slices were cut using a Leica VT1200S vibratome in ice-cold oxygenated (95\% O2 and 5\% CO2) mACSF. Slices recovered at room temperature for at least $1 \mathrm{~h}$ in artificial cerebrospinal fluid (ACSF) containing the following (in $\mathrm{mM}$ ): $126 \mathrm{NaCl}, 3.5 \mathrm{KCl}$, $1.25 \mathrm{NaH} 2 \mathrm{PO} 4,26 \mathrm{NaHCO}, 1.3 \mathrm{MgCl}$, 2.0 CaCl2, and $10 \mathrm{D}$-glucose. Both cutting solution and ACSF were between 290 mOsm and 310 mOsm. All solutions were equilibrated with $95 \%$ $\mathrm{O} 2$ and $5 \% \mathrm{CO} 2, \mathrm{pH} 7.4$.

fEPSP Recordings Acute slices were individually transferred to a recording chamber maintained at $30-32^{\circ} \mathrm{C}$ and continuously perfused $(2 \mathrm{ml} / \mathrm{min})$ with oxygenated ACSF. Field excitatory postsynaptic potential (fEPSP) were made in the lucidum of CA3 area with glass electrodes (2-3 M $\Omega$; filled with normal ACSF) using a DAM-80 amplifier (low filter, $1 \mathrm{~Hz}$; high pass filter, $3 \mathrm{KHz}$; World Precision Instruments, Sarasota, FL). Mossy fiber-mediated fEPSP were evoked by weak electrical stimulations performed via a bipolar NiCh electrode (NI-0.7F, Phymep, Paris) positioned in the lucidum of CA3 area; the stimulus intensity, pulse duration, and frequency were around $30 \mathrm{~V}, 25 \mu \mathrm{s}$, and $0.1 \mathrm{~Hz}$, respectively. Data were digitized with a Digidata 1440A (Molecular Devices) to a PC, and acquired using Clampex 10.1 software (PClamp, Molecular Devices). Signals were analyzed off-line using Clampfit 10.1 (Molecular Devices). LTP was induced by tetanus at $25 \mathrm{~Hz}$ during $5 \mathrm{~s}$; D-APV (D-2-amino-5phosphonovalerate, $40 \mu \mathrm{M}$ ) was included in ACSF during the tetanus to eliminate contamination of MF-CA3 LTP with NMDA receptor-dependent component. At the end of each experiment, $2 \mu \mathrm{M}$ DCG-IV, a group II mGluR agonist, was bath applied to confirm the mossy fiber synaptic origin of fEPSP recorded in CA3. The magnitude of long-term plasticity was determined by comparing baseline-averaged responses before induction with the last $10 \mathrm{~min}$ of the experiment. Example traces are averages of at least 30 consecutive sweeps taken from a single representative experiment. 


\section{The Hipposeq analysis}

The Hipposeq data, analysis, and website were obtained through collaboration between the Spruston Laboratory (Mark Cembrowski, Nelson Spruston), NeuroSeq (Lihua Wang, Erina Hana, Ken Sugino, Andrew Lemire), and Scientific Computing (Jody Clements, Jonathan Epstein) at the Janelia Research Campus of the Howard Hughes Medical Institute.

Statistical analysis All data are shown as means \pm SEM. Statistics were performed using IgorPro (Wavemetrics), and statistical significance was determined by the Student's test (two tailed distribution, paired) unless otherwise stated.

\section{Reagents}

Stock solutions were prepared in water or DMSO, depending on the manufacturers' recommendation, and stored at $-20^{\circ} \mathrm{C}$. Upon experimentation, reagents were bath applied following dilution into ACSF (1/1000). D-APV and DCGIV were purchased from Tocris Bioscience. Salts for making cutting solution and ACSF were purchased from Sigma.

\section{References}

Smith, D. J. et al. Functional screening of $2 \mathrm{Mb}$ of human chromosome 21q22.2 in transgenic mice implicates minibrain in learning defects associated with Down syndrome. Nat. Genet. 16, 28-36 (1997).

Lepagnol-Bestel, A.-M. et al. DYRK1A interacts with the REST/NRSF-SWI/SNF chromatin remodelling complex to deregulate gene clusters involved in the neuronal phenotypic traits of Down syndrome. Hum. Mol. Genet. 18, 1405-1414 (2009). 


\section{FIGURES}

\section{Figure 1. Interaction of HSA21 DYRK1A with chromatin remodelers}

A. Schematic representation of DYRK1A interaction with EP300 and CREBBP. B. HEK293 cells were immunoprecipitated (IP) using anti-EP300 and anti-CREBBP antibodies and using anti-IgG antiboby as a negative control. The input and precipitated fractions were analyzed by western blot using anti-Ep300, anti-Dyrk1a and anti-Crebbp antibodies. The arrows indicate proteic bands at the expected size. Note that no cross-reaction was found with the IgGs.

C-D. In situ proximity ligation assays (PLA) on primary cortical neurons fixed at DIC7 (red fluorescence) using anti-Dyrk1a and anti-Ep300 or anti-Crebbp, anti-Smarca2 and anti-Ep300 or anti-Crebbp antibodies. Nuclear bodies were labelled using Topro3 staining (blue fluorescence). Mean interaction point numbers were calculated in nuclear body of 45 to 89 cortical neurons at DIC7 (from 3 to 5 different embryos per genotype). PLA using anti-Ep300 and anti-Fibrillarin antibodies were performed as a negative control and no difference was shown between transgenic $189 \mathrm{~N} 3$ and WT cortical neurons. Scale bars $=10 \mu \mathrm{m} .{ }^{*} p<0.05$; ${ }^{* * *}$ $p<0.0005$. E. False-color image of ISH from Allen Brain Atlas showing Dyrk1a, Ep300 and Crebbp transcript expression in adult mouse hippocampus. Scale bar $=100 \mu \mathrm{m}$.

Figure 2. Presynaptic protein expression in the adult 152F7 mouse hippocampus as compared to control mice.

A. Schematic representation of molecules involved in glutamate release from presynaptic vesicles.

B. Western blotting of Rim1, Rab3a, Munc13-1 and Synapsin 2A proteins from hippocampus extracts of wild-type and 152F7 transgenic mice. The amounts of protein were normalised to actin levels. Four to five hippocampi were pooled. There was a decrease in the amount of Synapsin 2A in the 152F7 transgenic mice (152F7) as compared to the wild type (WT).

C \& D. Laser-assisted microdissection of the three subregions of P21 mouse hippocampus stained with toluidine blue (C). Scale bar $=200 \mu \mathrm{m}$. From Laser-assisted microdissection of the three subregions of P21 mouse hippocampus, Rims1, Syn2, Rab3a and Munc13-1 transcripts are down regulated in the $D G$ and $C A 3$ hippocampal subregions of juvenile transgenic $152 \mathrm{~F} 7$ mice compared to their WT siblings as shown by Q-RT-PCR analysis (D). Scale bar $=1 \mathrm{~mm} .{ }^{*} p<0.01^{* *} p<0.001 .{ }^{* * *} p<0.0001$

Figure 3. Presynaptic LTP between Dentate Gyrus mossy fibers and CA3 impaired in both adult 152F7 and 189N3 mouse hippocampus as compared to control mice.

A. Example of a temporal trace of mossy fiber LTP in a wild type mouse. Mossy fiber LTP was induced by a single tetanus of $25 \mathrm{~Hz}$ (for $5 \mathrm{~s}$, black arrow) in the presence of $50 \mu \mathrm{M}$ of DAP 5 . 
NBQX was applied at the end to obtain information concerning the fiber volley. Inset: example traces of a baseline fEPSP (left) and fEPSP after LTP (right).

B. Summary of mossy fiber LTP in transgenic 152F7 compared to WT mice, showing LTP induction defect in the DS mouse model. Data are means+/ SEM and calculated from 4 different mice with 3 slices per mouse for both genotypes.

C. D. Summary of mossy fiber LTP in transgenic $189 \mathrm{~N} 3$ compared to WT mice (C). Average fEPSP amplitude 20 min after LTP induction in WT versus 189N3 mice, showing LTP induction defect in the DS mouse model (D). Recording were performed on 4 to 6 mice in each genotypes. Data are means+/ SEM.

\section{Supplementary Figure 1. Schematic representation of the human YAC 152F7 transgene and of the mouse BAC 189 N3 transgene.}

We present here the genomic region of the human YAC 152F7 from the hg38 release (UCSC genome browser).

Note that human YAC 152F7 transgene (570 kb) involves a copy of six hChr21 genes: RIPPLY3, PIGP, TTC3, DSCR9, VPS26C and DYRK1A respectively.

In contrast, the mouse BAC 189N3 transgene consists of a $152 \mathrm{~kb}$ containing only the whole mouse Dyrk1a gene with a $6 \mathrm{~kb}$ flanking fragment on the 5 ' side and a $19 \mathrm{~kb}$ flanking fragment on the 3، (189N3 BAC clone from Research Genetics).

Supplementary Figure 2. Schematic representation of the mouse genomic region syntenic of the human YAC 152F7 transgene and that includes the mouse BAC 189N3 transgene.

We present here the mouse syntenic genomic region of the human YAC 152F7 (Mouse Dec. 2011 (GRCm38/mm10 release) (UCSC genome browser).

The YAC 152F7 transgene (570 kb) involves a copy of six hChr21 genes: RIPPLY3, PIGP, TTC3, DSCR9, VPS26C and DYRK1A respectively.

In contrast, the syntenic mouse region displays orthologous genes of RIPPLY3, PIGP, TTC3, DSCR9, VPS26C and DYRK1A in a more compact region of $\sim 300 \mathrm{~kb}$. DSCR9 is a primatespecific gene (Takamatsu et al., 2002).

\section{Supplementary Figure 3. Nuclear protein-protein interactions and deregulation of interaction network in transgenic mouse models.}

A. In situ proximity ligation assays PLA on primary cortical neurons fixed at DIC7 (red fluorescence) using anti-Ep300 and anti-Smarca2 antibodies, anti-Crebbp and anti-Smarca2 antibodies, anti-Smarca4 and anti-Smarca2 antibodies and anti-Ep300 and anti-Fibrillarin antibodies as a negative control. Nuclear bodies were labelled using Topro3 staining (blue 
fluorescence). Mean interaction point numbers were calculated in nuclear body of at least 48 cortical neurons at DIC7.

B. In situ proximity ligation assays PLA on transgenic 189N3 and WT primary cortical neurons fixed at DIC7 (red fluorescence) using anti-Baf155 and anti-Smarca4 antibodies. Nuclear bodies were labelled using Topro3 staining (blue fluorescence). Mean interaction point numbers were calculated in nuclear body of at least 48 cortical neurons at DIC7.

Scale bars $=10 \mu \mathrm{m} .{ }^{* \star *} p<0.0005$

\section{Supplementary Figure 4. Quantification of Rims1 RNA in WT and 152F7 hippocampus using quantitative In Situ Hybridation}

False-color image of antisense Rims1 RNA Q-ISH of hippocampus from juvenile P21 WT and $152 \mathrm{~F} 7$ mice. Q-ISH was performed using $3 \mathrm{H}$ radioactive probes for Rims1. Q-ISH quantification indicates a significant down-regulation of Rims1 in the three subregions of the 152F7 mouse hippocampus

\section{Supplementary Figure 5. Comparison of transcripts encoding presynaptic proteins using either Q-RT PCR from laser assisted microdissection or RNAseq single cell transcriptomics}

A. Regions of interest from which RNAseq single cell transcriptomics was performed in population(s) of excitatory cells is indicated (The Hipposeq data obtained from Janelia Farm website) (Cembrowski et al., 2016)

B. Comparison of our data from Laser-assisted microdissection of the three subregions of P21 mouse hippocampus of juvenile transgenic 152F7 mice compared to their WT siblings (see Figure 3D) and RNAseq data for Rims1, Syn2, Rab3a and Munc13-1 transcripts.

Scale bar $=1 \mathrm{~mm} .{ }^{*} p<0.01^{* *} p<0.001 .{ }^{* * *} p<0.0001$ 
A

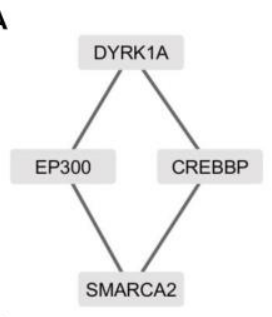

C DYRK1A DYRK1A

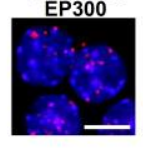

E

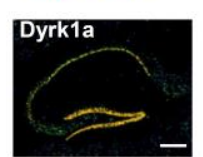

B

$28^{30^{\circ}}+22^{19^{\circ}}$

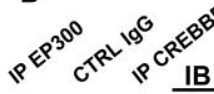

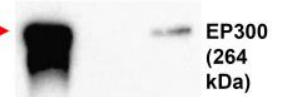

kDa)

DYRK1A $(85 \mathrm{kDa})$

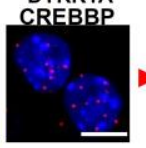

Ep300

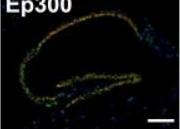

D
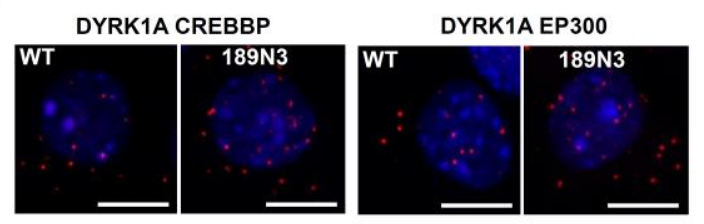

PLA per $\mu \mathrm{m}^{2}$ of nucleus

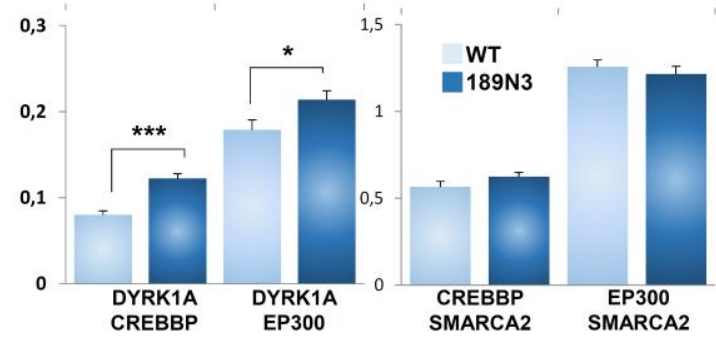

Figure 1 

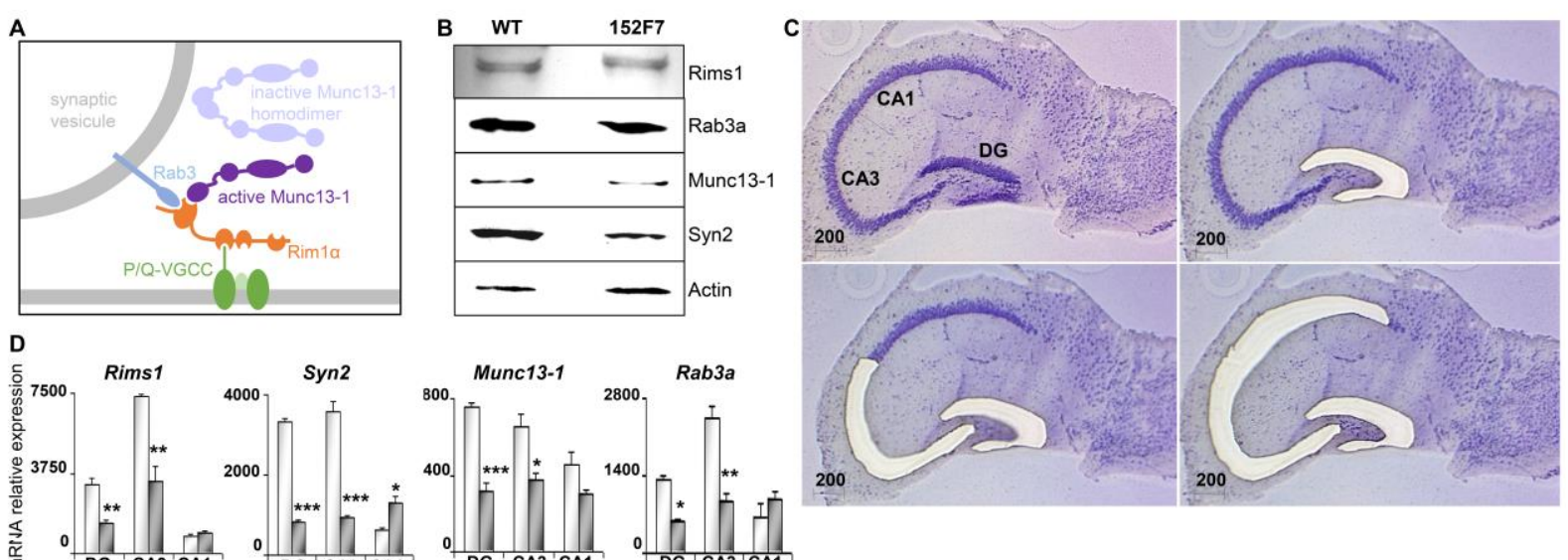

Figure 2 
Figure 3

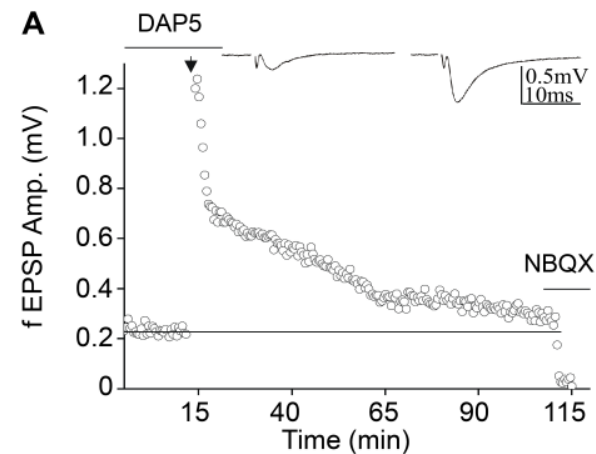

C

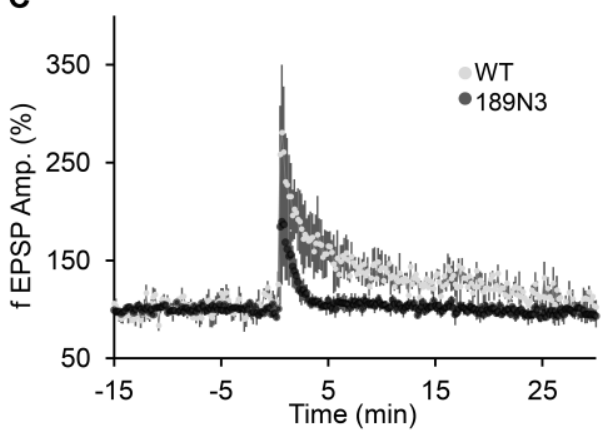

B

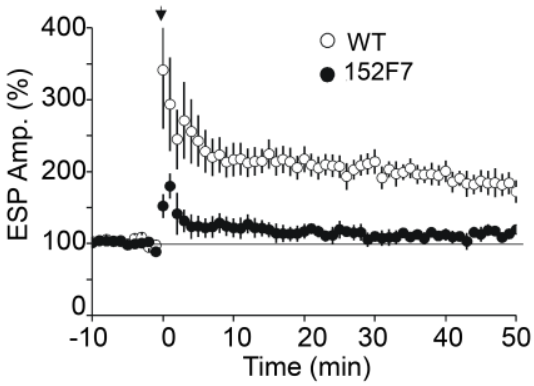

D

WT 189N3

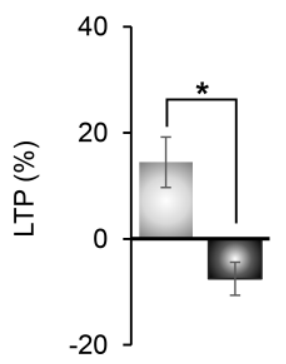

